\title{
Le siècle de Marie de Médicis, sous la direction de M. Fumaroli
}

\section{Sergio Poli}

\section{Q OpenEdition}

1 Journals

\section{Edizione digitale}

URL: https://journals.openedition.org/studifrancesi/40542

DOI: 10.4000/studifrancesi.40542

ISSN: 2421-5856

\section{Editore}

Rosenberg \& Sellier

\section{Edizione cartacea}

Data di pubblicazione: 1 juillet 2004

Paginazione: 177-178

ISSN: 0039-2944

\section{Notizia bibliografica digitale}

Sergio Poli, «Le siècle de Marie de Médicis, sous la direction de M. Fumaroli», Studi Francesi [Online], 142 (XLVIII | I) | 2004, online dal 30 novembre 2015, consultato il 09 septembre 2021. URL: http:// journals.openedition.org/studifrancesi/40542 ; DOI: https://doi.org/10.4000/studifrancesi.40542

Questo documento è stato generato automaticamente il 9 septembre 2021.

\section{(c) (i) (9)}

Studi Francesi è distribuita con Licenza Creative Commons Attribuzione - Non commerciale - Non opere derivate 4.0 Internazionale. 


\title{
Le siècle de Marie de Médicis, sous la direction de M. Fumaroli
}

\author{
Sergio Poli
}

\section{NOTIZIA}

AA. VV., Le siècle de Marie de Médicis, Actes du séminaire de la Chaire «Rhétorique et société en Europe (XVI ${ }^{\mathrm{e}}-\mathrm{XVII}{ }^{\mathrm{e}}$ siècle), sous la direction de M. Fumaroli, Alessandria, Ed. dall'Orso, 2003, pp. XVI-246.

1 Pubblicato a cura di Francesco Solinas e di Françoise Graziani, questo elegante volume in $-4^{\circ}$ costituisce anche un numero speciale della rivista «Franco-Italica», che, come sottolinea la fondatrice D. Dalla Valle nell'»Avvertenza», si dedica da dieci anni ad illustrare i diversi e molteplici rapporti che legano le due culture transalpine. Ad un orizzonte siffatto si adatta perfettamente il tema del seminario, incentrato attorno alla figura di Maria de' Medici, la quale, come l'ava Caterina, incarna più di ogni altra non solo l'intensità ed il livello delle relazioni tra i due paesi, ma anche l'ambigua contraddittorietà che storicamente le distingue e che mescola, da parte francese, positiva ammirazione a permanenti stereotipi negativi. Da quest'ultimo punto di vista il seminario porta un prezioso contributo alla «riabilitazione» ed alla valorizzazione dell'operato di Maria, giungendo, come afferma M. Fumaroli nella dotta introduzione (pp. XIII-XVI), a definire «cette période relativement mal connue des lettres et des arts français» come «le siècle de Marie».

2 Il volume si divide in tre sezioni, che ampliano via via il campo dell'analisi dalla figura della regina agli orizzonti delle influenze interculturali. Le prima è dedicata alla Royne Florentine, e riguarda la formazione, la vita e le relazioni politiche della Medici inquadrandole nella politica dinastica della famiglia. Si apre, molto opportunamente, con la presentazione del Ritratto di Maria de' Medici giovane (pp. 3-12), che offre a F. SOLINAs la possibilità non solo di presentarci l'opera del ritrattista mantovano Pietro Fachetti, ma di introdurci nel vivo della politica medicea, delle sue strategie europee e 
del suo uso strumentale delle arti. Proprio quest'ultimo aspetto è al centro del contributo di M. CHAPEL, che ripercorre per noi The Artistic education of Maria de' Medici (pp. 13-25), sottolineando il gusto «barocco» della Regina e l'importanza della sua influenza sulla pittura francese; mentre S. MAMONE (L'eredità medici, pp. 27-40) allarga il quadro a tutto un patrimonio culturale, estetico e ideologico che, grazie a Maria, non solo regina di Francia, ma anche madre e parente stretta di sovrani europei, si diffonde a livello continentale. Poteva restare estranea a tutto questo la dimensione religiosa? L'avvento di Maria al trono di Francia rappresenta, per la monarchia borbonica, nata da guerre confessionali, non solo un affare economico, ma anche una scelta politica per ancorare il paese al mondo cattolico; una scelta che Maria porterà ad estreme conseguenze «ultramontane» (B. BARBICHE Marie et le saint-Siège, pp. 41-49) e che non fu forse estranea ad un declino illustrato, da diversi punti di vista, negli ultimi contributi della sezione. o. RANUM ci introduce infatti nella rivalità tra Maria e Luigi XIII (Richelieu et la "Querelle de la mère et du fils», pp. 57-65), fondamentale per comprendere come la sconfitta della madre ad opera del figlio segni anche il passaggio ad una concezione non familiare della monarchia; e C. NATIVEL ci porta ai festeggiamenti per L'entrée de marie de Médicis à Anvers. La fin du rêve italien, (pp. 67-78), che segnano l'esilio definitivo di Maria, illustrandone la portata metaforica e politica.

La seconda parte del volume si concentra tutta attorno a Marie et les artistes, ed esplora su più versanti rapporti fondamentali per l'evoluzione della pittura francese dell'epoca. P. BASSANI PACHT appunta la sua attenzione non tanto su «la période la plus glorieuse de l'école de Fontainebleau", ma sugli anni, più oscuri, della Reggenza (Marie de Médicis et ses artistes pp. 81-93), che pure segnò, in particolare con il ciclo di pitture dell'ala sud del Louvre, oggi in parte perduto, un'importante evoluzione estetica e contribuì all'elaborazione di una vera e propria leggenda di Maria; mentre N. SAINT FARE GARNOT, nel suo Philippe de Champaigne, peintre de Marie de Médicis (pp. 95-105), modificando in parte opinioni diffuse, illustra, grazie sia ad una documentazione di prima mano che ad un'attenta analisi iconografica, il «lien particulier qui unissait le peintre et son illustre commanditaire». Con L. PERICOLO entriamo per un lungo percorso nel palazzo del Luxembourg: il suo articolo ci introduce infatti nella sua "architecture stupéfiante»e tra decorazioni in cui la mescolanza di sacro e profano, maschile e femminile, natura e artificio contribuisce a caratterizzare esteticamente «le siècle de Marie» come quello dell'»union des contraires» (Le palais riant d'une «femme forte»: M. de Médicis et le Luxembourg, pp. 107-118); quello di R. ConTINI ci illustra le pitture del «Cabinet Doré», gettando nuova luce tanto sul gruppo di artisti «minori» che vi collaborarono, quanto sui modi e sugli obiettivi della committenza (Pittori minori - ma veramente tali? - per il Cabinet Doré, pp. 119-124); mentre con M. WASCHEK ci fermiamo di fronte al «ciclo Medici» di Rubens (Le cycle Médicis comme discours panégyrique, pp. 125-136), la cui storia, grazie ad una fine ricostruzione delle circostanze che ne videro la creazione e delle vicende successive, ci permette di approfondire non solo l'intreccio dei rapporti che legano estetica, politica ed ideologia, ma anche la loro dinamica nel tempo. Quanto un simile intreccio sia fondamentale lo dimostrano anche i due ultimi articoli della sezione. Il primo, di I. CISERI, torna sul tema dei Ballets et carrousels: immagini simboliche nello spettacolo di corte (pp. 137-144), delle loro coreografie geometriche e celebrative attorno al principe cardine di un universo metaforico; il secondo, di A. BRESSON, ci mostra la convergenza di letteratura e arti nella glorificazione di Maria (La glorification littéraire et artistique de M. de Médicis: le rôle de Malherbe et celui de Peiresc, pp. 145-161), 
sottolineando in particolare il ruolo di Peiresc come collaboratore del mecenatismo della sovrana e come punto di raccordo tra gli intellettuali francesi ed europei.

La terza sezione, infine, ci sposta nettamente nel campo franco-italiano trattando del Dialogue des lettres italiennes et françaises. Teatro, poesia, mecenatismo e traduzione ne sono i necessari fili conduttori. Ecco, nel teatro, La dramaturgie de Giovan Battista Andreini pour Marie de Médicis (S. FERONE, pp. 165-173) riaffermare, dopo la morte di Concini ed il ritorno di Maria dall'esilio e su modi neoplatonici, il valore dello spettacolo come mezzo di governo sociale; per la poesia, inevitabile incontrare la figura centralissima del Marino, di cui il compianto G. Fulco analizza lettere dalla Francia, aggiungendo nuovi documenti alla corrispondenza già nota (La corrispondenza di G.B. Marino dalla Francia, pp. 175-185) e del quale F. GRAZIANI traccia l'influenza sui poeti d'oltralpe ( $L a$ langue des dieux: Marino et les jeunes poètes français, pp. 187-203), evidente soprattutto nel campo dell'idillio, con le imitazioni di Tristan, Saint-Amant, o Puget de la Serre. Quanto al mecenatismo, il dotto contributo di J.-P. CHAUVEAU, dedicato al Mécénat diu comte de Moret (pp. 203-211), ci offre, più che il ritratto di un personaggio, che morì giovanissimo (25 anni) e non poté quindi patrocinare a lungo le lettere francesi, quello dell'ambiente intellettuale degli anni '20; mentre F. LA BRASCA, con il suo Les traductions d'oeuvres italiennes et françaises sous la régence de Marie de Médicis (pp. 213-223), portando la questione delle influenze su un terreno estremamente concreto, ci regala un prezioso repertorio delle traduzioni di testi italiani che commenta con attenzione e che mostra quanto «la 'France italienne' (...) de la régence de Marie de Médicis existe donc de façon bien vivante» e quanto sia ricca e varia.

La bella Conclusione di Cecilia Rizza, come del resto l'introduzione di M. Fumaroli, non si limita a raccogliere con intelligenza le fila di un seminario che rappresenta un punto fermo negli studi in questo campo, ma, da specialista qual è del periodo, sa conferire all'insieme una prospettiva storica unitaria che integra con opportune citazioni. Essa chiude degnamente un volume che si raccomanda non solo per il suo contenuto critico, ma anche per le bellissime ed abbondanti riproduzioni di quadri e disegni, che da sole costituiscono un notevole motivo di interesse, nonché per le caratteristiche eleganti e curate della stampa. 